\title{
Myopieprogressionshemmung - Zusammenfassung der aktuellen Literatur
}

\author{
Clemens A. Strohmaier für die Kommission für Refraktion, Optometrie und Kontaktologie der Österreichischen \\ Ophthalmologischen Gesellschaft · Stefan Pieh für die Kommission für Refraktion, Optometrie und \\ Kontaktologie der Österreichischen Ophthalmologischen Gesellschaft
}

Eingegangen: 3. Juni 2019 / Angenommen: 15. Juni 2019 / Online publiziert: 2. Juli 2019

(C) Der/die Autor(en) 2019

\begin{abstract}
Zusammenfassung
Hintergrund Kurzsichtigkeit kann zwar mit optischen Hilfsmitteln korrigiert werden, erhöht jedoch auch bei geringer Ausprägung das Risiko für eine Sehbehinderung durch andere Augenkrankheiten im späteren Lebensalter. Insbesondere hohe Myopie sollte vermieden werden. Ziel der gegenwärtigen Arbeit ist es, die rezente Literatur zum Thema Myopieprogressionshemmung zusammenzufassen.

Material und Methode Es wurde eine Literatursuche mittels Medline/PubMed durchgeführt und die rezente Literatur zusammengefasst, wobei Metaanalysen und randomisiert kontrollierte Studien bevorzugt verwendet wurden, sofern verfügbar.

Resultate Zeit im Freien, pharmakologische Therapie mit Atropin (oder ähnlichen Substanzen) sowie optische Methoden (Bifokal/Gleitsichtbrille, Orthokeratologie, Multifokalkontaktlinsen sowie Kontaktlinsen mit hoher positiver sphärischer Aberration) sind wirksam zur Hemmung der Myopieprogression. Zeit im Freien ist zusätzlich wirksam in Bezug auf die Inzidenzhemmung. Unklar sind derzeit v. a. die optimale Dauer der Therapie sowie die Wirksamkeit in höheren Lebensjahren.

Schlussfolgerung Zur Myopieprogressionshemmung stehen aktuell wirksame Therapien zur Verfügung und sollten im klinischen Alltag in Erwägung gezogen werden.
\end{abstract}

PD Dr. C. A. Strohmaier, $\mathrm{PhD}(\bowtie)$

Universitätsklinik für Augenheilkunde und Optometrie, Kepler Universitätsklinikum GmbH, Johannes Kepler

Universität Linz, Krankenhausstr. 7a, 4020 Linz, Österreich

clemens.strohmaier@kepleruniklinikum.at

Univ. Prof. Dr. S. Pieh

Universitätsklinik für Augenheilkunde und Optometrie,

Medizinische Universität Wien, Wien, Österreich
Schlüsselwörter Kontaktlinsen · Atropin . Augenkrankheit · Netzhauterkrankung · Prävention

Inhibition of myopia progression-review of the current literature

\section{Summary}

Background Myopia can be corrected using optical aids but even in cases of low expression significantly increases the risk for visual impairment due to other eye diseases at higher ages. Especially high-grade myopia should be prevented. The purpose of the present study was to review the present literature concerning inhibition of myopia progression.

Material and methods An online literature search was performed using Medline/PubMed and a summary of relevant papers is presented. Meta-analyses and prospective randomized controlled trials were preferably used if available.

Results Time spent outdoors, pharmacological treatment with atropine (and similar substances) and optical methods (bifocal/varifocal glasses, orthokeratology, multifocal contact lenses as well as contact lenses with positive spherical aberration) have been shown to slow myopia progression. Time spent outdoors further reduces myopia incidence. The optimal duration of treatment as well as the efficacy at higher ages currently lacks evidence.

Conclusion Effective forms of treatment for inhibition of myopia progression are readily available and should be considered in clinical practice.

Keywords Contact lens - Atropine - Eye disease · Retinal disease $\cdot$ Prevention 
Myopie als alltäglicher augenärztlicher Vorstellungsgrund wurde lange nur bei hoher Ausprägung als Erkrankung angesehen. Verglichen mit anderen Risikofaktoren, wie z. B. Bluthochdruck für zerebralen Insult, führt jedoch bereits eine geringgradige Myopie zu einer deutlichen Risikoerhöhung hinsichtlich einer Netzhautabhebung und myoper Makulaerkrankungen [1]. Aus diesem Grund sollte die Entstehung und Progression der Myopie soweit als möglich verhindert werden.

In den letzten Jahren hat es hinsichtlich therapeutischer Möglichkeiten zur Progressionshemmung neue Erkenntnisse gegeben, die im Folgenden zusammengefasst werden sollen. Ziel dieser Arbeit ist, eine Übersicht wesentlicher Erkenntnisse für den klinischen Alltag zu liefern, für eine ausführlichere Literaturübersicht kann zum derzeitigen Zeitpunkt (2019) insbesondere auf die Zusammenfassungen des International Myopia Institutes verwiesen werden [2], abrufbar unter https://www.myopiainstitute.org/imiwhite-papers.html.

Für therapeutische Empfehlungen wurden primär prospektiv randomisierte kontrollierte Studien (entsprechend Evidenzgrad Ib) und Metaanalysen (Evidenzgrad Ia) berücksichtigt.

Wirksame Therapien können derzeit in 3 Gruppen eingeteilt werden [3]:

1. Zeit im Freien (Inzidenz- und Progressionshemmung),

2. pharmakologische Therapie - Atropin (Progressionshemmung),

3. optische Methoden (Progressionshemmung):

a. Bifokal-/Gleitsichtbrillen,

b. Multifokale Kontaktlinsen oder Kontaktlinsen mit hoher positiver sphärischer Aberration,

c. Orthokeratologie.

Diese Therapieansätze sollen im Folgenden vorgestellt werden. $\mathrm{Zu}$ beachten ist, dass die Mehrheit der Studien im asiatischen Raum durchgeführt wurde und mögliche Unterschiede zwischen Ethnien in Bezug auf die Entwicklung einer Myopie oder der Wirksamkeit der therapeutischen Intervention derzeit nicht genau untersucht sind. Arbeiten zeigen für einzelne AtropinDosierungen in kleineren Kohorten jedoch eine vergleichbare Wirksamkeit für eine europäische Population [4]. Auch für multifokale Kontaktlinsen entsprechen Daten aus Europa dem asiatischen Durchschnitt [5].

\section{Bestimmung der Refraktion zur Indikations- stellung}

Aufgrund der Akkommodation ist ausschließlich eine Refraktionsbestimmung in Zykloplegie zur Bestimmung einer myopen Ametropie im Kinder- und Jugendalter zu verwenden. Bezüglich Pharmaka zur Zykloplegie wird auf die entsprechenden Empfehlungen des Consilium Strabologicum Austriacum hingewie- sen. Die Diagnose einer Myopie wird ab -0,5 dpt gestellt, die Diagnose progrediente Myopie ab einer $\mathrm{Zu}-$ nahme von $0,5 \mathrm{dpt}$ pro Jahr.

Die Bestimmung der Achsenlänge ist derzeit in der klinischen Routine noch nicht weit verbreitet, stellt jedoch ein Instrument zur objektiven Bestimmung des Augenlängenwachstums dar. In beinahe allen Studien zur Myopieprogression liegen dafür gute Daten vor. Tideman et al. haben für eine kaukasische Kohorte Normwerte für jede Altersstufe publiziert, die eine Risikoabschätzung bezüglich der Entwicklung einer Myopie ermöglichen [6].

\section{Zeit im Freien}

Zeit im Freien schützt sowohl vor dem Auftreten einer Myopie (Inzidenzhemmung) als auch vor der Progression einer bereits bestehen Myopie (Progressionshemmung) $[7,8]$.

Die Dosis-/Wirkungsbeziehung schwankt zwischen den Studien. Als Richtwert kann man bei einem Aufenthalt im Freien von $7 \mathrm{~h}$ pro Woche von einer um grob $50 \%$ verringerten Inzidenz ausgehen [9].

Für die Progressionshemmung war die Datenlage lange unsicher. In einer großen Studie von 2018 konnte ein positiver Effekt gezeigt werden. Schulkinder wurden ermutigt, mehr als $11 \mathrm{~h} /$ Woche im Freien zu verbringen, wodurch eine annähernd $30 \%$ ige Hemmung der Myopieprogression erzielt werden konnte[8].

Der Myopie-hemmende Effekt kommt durch die erhöhte Lichtintensität zustande, wobei die benötigte Intensität bereits an einem bewölkten Tag erreicht wird (>1000lx). Unklar ist jedoch die benötigte Gesamtdosis (Intensität $\times$ Zeitdauer).

\section{Atropin}

Topische Anwendung von $1 \%$ igem Atropin ist schon lange als wirksame Therapie zur Hemmung der Myopieprogression bekannt, wurde jedoch aufgrund der starken Nebenwirkungen kaum eingesetzt.

Aus den ATOM (Atropine for the treatment of Myopia) Trials wurden einige wichtige Erkenntnisse gewonnen, die Atropin in niedrigerer Konzentration derzeit als sehr vielversprechend erscheinen lassen [10]:

- Atropin in 0,01 \%iger Dosierung hemmt die Progression in Bezug auf das sphärische Äquivalent.

- Atropin 0,01\% führt zu keiner subjektiven visuellen Beeinträchtigung (Blendung, Nahsehleistung).

- Der Rebound Effekt nach Therapiestopp ist am geringsten ausgeprägt (s. unten).

- Gutes Ansprechen bei Wiederbeginn der Therapie.

$\mathrm{Zu}$ beachten ist, dass eine wirksame Hemmung des Achsenlängenwachstums derzeit nicht gezeigt werden konnte. Über die Gründe dafür kann keine verlässliche Aussage getroffen werden. Eine mögliche Erklärung ist jedoch das Studiendesign der ATOM2-Studie. Klarheit 
bezüglich des Effekts auf die Achsenlänge sollten die Follow-up-Studien ATOM3 und MTS1 (NCT03140358 und NCT03334253) bringen.

Die LAMP-Studie (Low Concentration Atropine for Myopia Progression) untersuchte Konzentrationen zwischen 0,01 und $0,05 \%$ und konnte für $0,05 \%$ iges Atropin 1-mal täglich einen signifikanten hemmenden Effekt auf Achsenlänge und sphärisches Äquivalent zeigen [11]. Die Nebenwirkungen wie subjektives Blendungsgefühl und verminderter Nahvisus unterschieden sich dabei nicht signifikant von der Placebound der 0,01\%-Gruppe. Derzeit sind jedoch nur Einjahresdaten veröffentlicht.

\section{Optische Methoden}

Wirksame optische Methoden sind:

- Bifokal- und Gleitsichtbrillen,

- Kontaktlinsen - multifokal bzw. mit positiver sphärischer Aberration,

- Orthokeratologie (OrthoK).

Brillen sind im Durchschnitt schwächer wirksam als OrthoK- und Kontaktlinsen. Die Studienergebnisse weisen eine höhere Variabilität in Bezug auf die Wirksamkeit auf [3, 12], können bei Kontraindikationen oder Patientenwunsch jedoch eine Alternative sein.

Eine gängige Additionsstärke in den bisherigen Studien ist $+2,0 \mathrm{dpt}$. Es gibt bis dato noch keine systematische Untersuchung zum Vergleich verschiedener Additionsstärken oder Linsendesigns, Ergebnisse sind jedoch in näherer Zukunft zu erwarten [13].

Als nicht wirksam zeigten sich monofokale formstabile Kontaktlinsen und die Unterkorrektur mit monofokalen Gläsern oder Kontaktlinsen. Es gibt Hinweise, dass eine Unterkorrektur die Myopieprogression sogar fördern könnte [14].

\section{Beginn und Dauer der Therapie}

In der Anamnese ist v. a. eine Myopie der Eltern relevant, wobei 2 myope Elternteile das Risiko für das Auftreten von Kurzsichtigkeit um etwa das Dreifache erhöhen [15]. Unklar ist, ob es sich um einen rein genetischen Effekt handelt, da Kinder myoper Eltern beispielsweise weniger Zeit im Freien verbringen [16].

Ein weiterer, wesentlicher Risikofaktor ist ein junges Lebensalter bei Auftreten der Myopie, da dadurch insbesondere die Entwicklung einer hohen Myopie wahrscheinlicher wird [17].

In Erwägung gezogen werden kann eine progressionshemmende Therapie bei Diagnosestellung einer Myopie (stärker als 0,5 dpt sphärisches Äquivalent) und einer Progression über 0,5 dpt/Jahr (Bestimmung unter Zykloplegie). Ab $1 \mathrm{dpt} / \mathrm{Jahr}$ spricht man von schneller Progression.

Der Einsatz einer progressionshemmenden Therapie ist nach derzeitiger Datenlage in einem früheren Lebensalter, als man aus der täglichen Routine vermu-

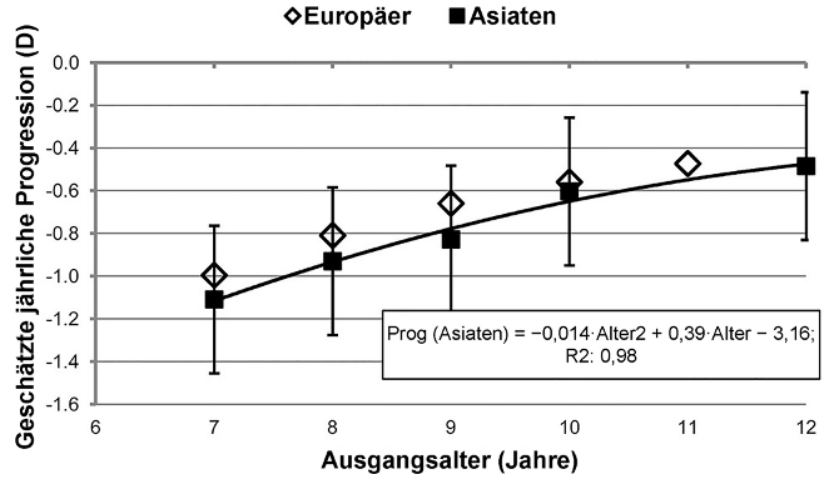

Abb. 1 Durchschnittliche Myopieprogressionsrate in der Kindheit (Aus [22]. Übersetzung und Abdruck mit freundlicher Genehmigung von Wolters Kluwer Health, Inc.)

ten würde, sinnvoll. Für OrthoK-Linsen konnte gezeigt werden, dass ein früher Anwendungsbeginn ab dem 6. Lebensjahr (LJ) wesentlich ist, da ihre therapeutische Wirksamkeit mit dem Lebensalter abnimmt. Bereits nach dem 14. LJ konnte in diesen Kohorten keine Progressionshemmung mehr nachgewiesen werden $[18,19]$.

Auch für die topische Atropingabe wird ein äquivalentes therapeutisches Zeitfenster vermutet, bisher jedoch nicht systematisch untersucht. Nach aktueller Datenlage ist bei progredienter Myopie (Zunahme von mehr als 0,5 dpt/Jahr, Bestimmung in Zykloplegie) ein Therapiebeginn ab dem 6 . LJ sinnvoll. Zu beachten ist, dass sich diese Angabe aus dem Design der aktuellen Studien ergibt - es kann individuell durchaus sinnvoll sein, bereits früher mit einer progressionshemmenden Therapie zu beginnen. Ein Beispiel dafür wäre ein Kind mit 2 hochmyopen Eltern und ungewöhnlich niedriger Hyperopie oder Emmetropie in einem jungen Lebensalter.

Zur Dauer der Therapie gibt es derzeit keine verlässlichen Daten, es ist jedoch, sowohl für Atropin als auch für optische Methoden, ein „Rebound-Effekt“ gezeigt worden. Nach Absetzen der Therapie zeigte sich ein beschleunigtes Achsenlängenwachstum. Wird ein solcher Effekt beobachtet, ist ein Wiederbeginn der Therapie sinnvoll und zeigt meist ein gutes Ansprechen [10, 20].

Derzeit noch nicht geklärt ist die Wirksamkeit sämtlicher progressionshemmender Methoden bei jenen Patienten, die nach dem 16. LJ eine starke Progression zeigen. In einer Langzeitkohorte zeigen etwa $5 \%$ der Teilnehmer keine Stabilisierung von Achsenlänge und sphärischem Äquivalent bis zum 30. LJ [21].

\section{Wirkung der Therapie}

Nach Therapiebeginn wird eine engmaschige Kontrolle des Patienten von 6 bis 12 Monaten, adaptiert an das individuelle Risiko, empfohlen. Zur Beurteilung der Wirkung kann je nach Alter bei Therapiebeginn und Vorbefunden die bisherige Progressionsrate her- 
Tab. 1 Überblick über die Effektstärke von verschiedenen Therapien zur Behandlung der progredienten Myopie, vereinfacht nach [3]

\begin{tabular}{|c|c|c|c|c|}
\hline & Ineffektiv & Schwach wirksam & Moderat wirksam & Stark wirksam \\
\hline Atropin $0,5-1,0 \%$ & - & - & - & $\begin{array}{l}\text { R: } 0,68 \\
\text { AL: }-0,21\end{array}$ \\
\hline Atropin $0,1 \%$ & - & - & - & $\begin{array}{l}\text { R: } 0,53 \\
\text { AL: }-0,21\end{array}$ \\
\hline Atropin $0,01 \%$ & - & - & AL: $-0,15$ & R: 0,53 \\
\hline $\begin{array}{l}\text { Kontaktlinsen (multifokal oder positive sphäri- } \\
\text { sche Abberation) }\end{array}$ & - & R: 0,21 & $\mathrm{AL}:-0,11$ & - \\
\hline Orthokeratologie & - & - & AL: $-0,15$ & - \\
\hline Gleitsichtbrille & - & $\begin{array}{l}\text { R: } 0,14 \\
\text { AL: }-0,04\end{array}$ & - & - \\
\hline Bifokalbrille & - & $\begin{array}{l}\text { R: } 0,09 \\
\text { AL: }-0,06\end{array}$ & - & - \\
\hline Formstabile Kontaktlinse monofokal & Kein signifikanter Effekt & - & - & - \\
\hline Unterkorrektur & Kein signifikanter Effekt & - & - & - \\
\hline Monofokalkorrektur & Vergleichsgruppe & - & - & - \\
\hline
\end{tabular}

angezogen werden. Zu beachten ist jedoch die Änderung der Progression je nach Alter. Ohne Vorbefunde sind Mittelwerte aus publizierten Metaanalysen hilfreich (Abb. 1; [22]).

Bei Nichtansprechen kann ein Wechsel der Therapie sinnvoll sein, allerdings gibt es dazu keine systematischen Untersuchungen.

Die erzielbare Progressionshemmung kann aus Tab. 1 entnommen werden. Dabei handelt es sich um eine rezente Metaanalyse zum Vergleich der Interventionen bei progredienter Myopie [3].

Funding Open access funding provided by Kepler Universitätsklinikum Linz.

Interessenkonflikt C.A. Strohmaier und S. Pieh geben an, dass kein Interessenkonflikt besteht.

Open Access Dieser Artikel wird unter der Creative Commons Namensnennung 4.0 International Lizenz (http:// creativecommons.org/licenses/by/4.0/deed.de) veröffentlicht, welche die Nutzung, Vervielfältigung, Bearbeitung, Verbreitung und Wiedergabe in jeglichem Medium und Format erlaubt, sofern Sie den/die ursprünglichen Autor(en) und die Quelle ordnungsgemäß nennen, einen Link zur Creative Commons Lizenz beifügen und angeben, ob Änderungen vorgenommen wurden.

\section{Literatur}

1. Flitcroft DI. The complexinteractions of retinal, optical and environmental factors in myopia aetiology. Prog Retin Eye Res. 2012;31:622-60.

2. Wolffsohn JS, Flitcroft DI, Gifford KL, Jong M, Jones L, Klaver CCW, et al. IMI - myopia control reports overview and introduction. Invest Opthalmol Vis Sci. 2019;60:M1.

3. Huang J, Wen D, Wang Q, McAlinden C, Flitcroft I, Chen H, et al. Efficacy comparison of 16 interventions for myopia control in children. Ophthalmology. 2016;123:697-708.
4. Polling JR, Kok RGW, Tideman JWL, Meskat B, Klaver CCW. Effectiveness study of atropine for progressive myopia in Europeans. Eye. 2016;30:998-1004.

5. Ruiz-PomedaA, Pérez-SánchezB, Valls I, Prieto-Garrido FL, Gutiérrez-Ortega R, Villa-Collar C. MiSight Assessment Study Spain (MASS). A 2-year randomized clinical trial. Graefes Arch Clin Exp Ophthalmol. 2018;256:1011-21.

6. Tideman JWL, Polling JR, Vingerling JR, Jaddoe VWV, Williams C, Guggenheim JA, et al. Axial length growth and the risk of developing myopia in European children. Acta Ophthalmol. 2018;96:301-9.

7. Sherwin JC, Reacher MH, Keogh RH, Khawaja AP, Mackey DA, Foster PJ. The association between time spent outdoors and myopia in children and adolescents. Ophthalmology. 2012;119:2141-51.

8. Wu P-C, Chen C-T, Lin K-K, Sun C-C, Kuo C-N, Huang H-M, et al. Myopia prevention and outdoor light intensity in a school-based cluster randomized trial. Ophthalmology. 2018;125:1239-1250

9. Xiong S, Sankaridurg P, Naduvilath T, Zang J, Zou H, Zhu J, et al. Time spent in outdoor activities in relation to myopia prevention and control: a meta-analysis and systematic review. Acta Ophthalmol. 2017;95:551-66.

10. Chia A, Lu QS, Tan D. Five-year clinical trial on atropine for the treatment of myopia 2 myopia control with atropine 0.01 \% Eyedrops. Ophthalmology. 2016;123:391-9.

11. Yam JC, Jiang Y, Tang SM, Law AKP, Chan JJ, Wong E, et al. Low-concentration atropine for myopia progression (LAMP) study. Ophthalmology. 2019;126:113-24.

12. Smith EL. Optical treatment strategies to slow myopia progression: Effects of the visual extent of the optical treatmentzone. Exp Eye Res. 2013;114:77-88.

13. Walline JJ, Gaume Giannoni A, Sinnott LT, Chandler MA, Huang J, Mutti DO, et al. A randomized trial of soft Multifocal contact lenses for myopia control. Optom Vis Sci. 2017;94:856-66.

14. Chung K, Mohidin N, O'Leary DJ. Undercorrection of myopia enhances rather than inhibits myopia progression. Vision Res. 2002;42:2555-9.

15. Mutti DO, Mitchell GL, Moeschberger ML, Jones LA, Zadnik K. Parental myopia, near work, school achievement, 
and children's refractive error. Invest Ophthalmol Vis Sci. 2002;43:3633-40.

16. Rose KA, Morgan IG, Ip J, Kifley A, Huynh S, Smith W, et al. Outdoor activity reduces the prevalence of myopia in children. Ophthalmology.2008;115:1279-85.

17. Price H, Allen PM, Radhakrishnan H, Calver R, Rae S, Theagarayan B, et al. The cambridge anti-myopia study. Optom Vis Sci. 2013;90:1274-83.

18. Hiraoka T, Kakita T, Okamoto F, Takahashi H, Oshika T. Long-term effect of overnight Orthokeratology on axial length elongation in childhood myopia: a 5-year follow-up study. Invest Opthalmol Vis Sci. 2012;53:3913.

19. Cho P, Cheung S-W. Retardation of myopia in Orthokeratology (ROMIO) study: a 2-year randomized clinical trial. Invest OpthalmolVis Sci. 2012;53:7077.
20. Cho P, Cheung SW. Discontinuation of orthokeratology on eyeball elongation (DOEE). Cont Lens Anterior Eye. 2017;40:82-7.

21. Hou W, Norton TT, Hyman L, Gwiazda J, COMET Group. Axial elongation in myopic children and its association with myopia progression in the correction of myopia evaluation trial. Eye Cont Lens Sci Clin Pract. 2018;44:248-59.

22. Donovan L, Sankaridurg P, Ho A, Naduvilath T, Smith EL, Holden AB. Myopia progression rates in Urban children wearing single-vision spectacles. Optom Vis Sci. 2012;89:27-32.

Hinweis des Verlags Der Verlag bleibt in Hinblick auf geografische Zuordnungen und Gebietsbezeichnungen in veröffentlichten Karten und Institutsadressen neutral. 